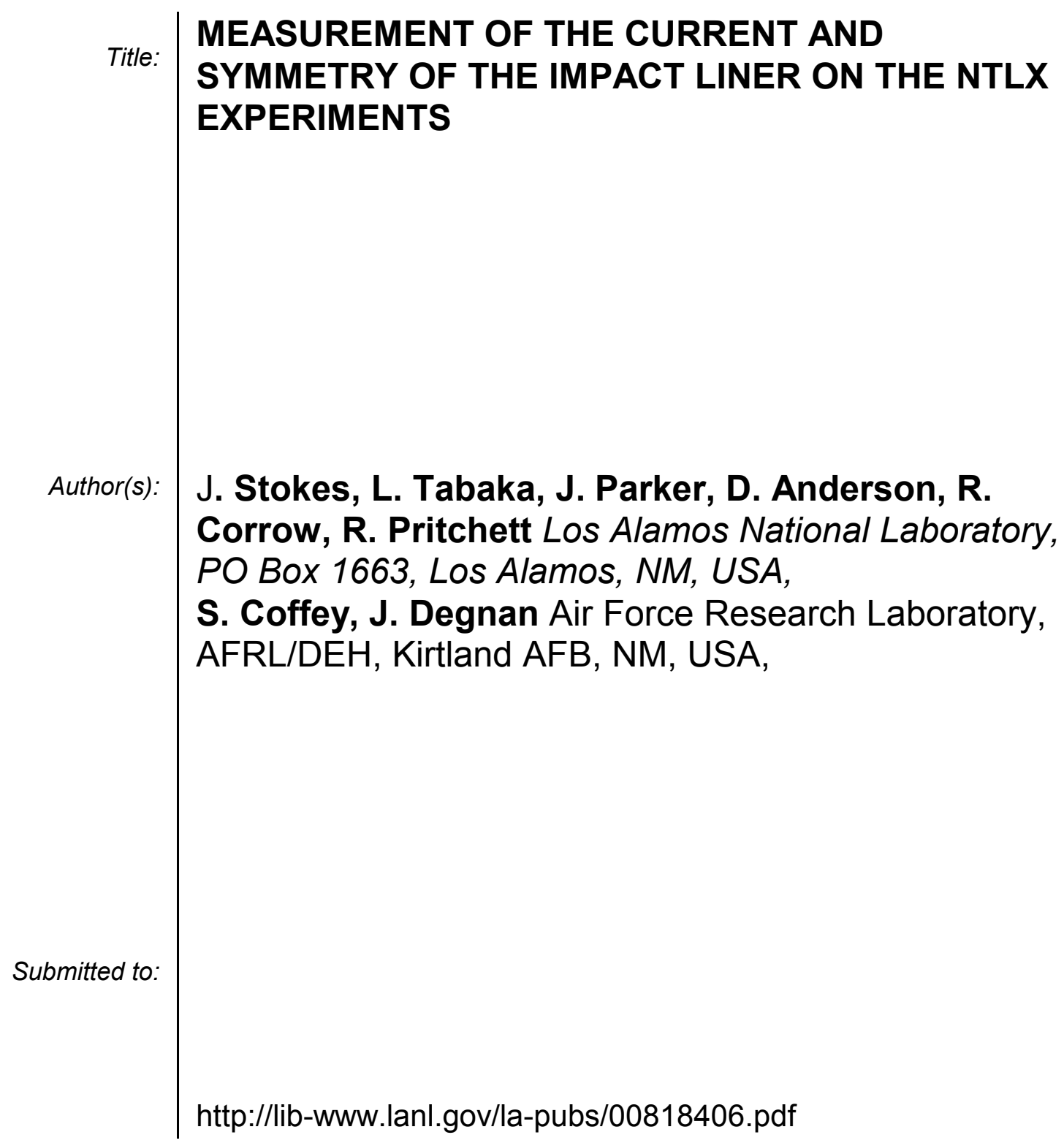




\title{
MEASUREMENT OF THE CURRENT AND SYMMETRY OF THE IMPACT LINER ON THE NTLX EXPERIMENTS*
}

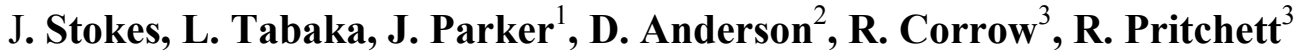 \\ Los Alamos National Laboratory, PO Box 1663, Los Alamos, NM, USA, \\ S. Coffey ${ }^{4}$, J. Degnan \\ Air Force Research Laboratory, AFRL/DEH, Kirtland AFB, NM, USA,
}

\begin{abstract}
A series of four liner implosion experiments, denoted the Near Tern Liner Experiments (NTLX) was recently conducted on the Shiva Star capacitor bank at the Air Force Research Laboratory (AFRL). Measurement of the driving currents in these experiments is required for postshot analysis of the liner implosion and experiments conducted in the target cylinder. A Faraday rotation measurement was fielded on Shiva Star to measure the current and compare with the current measured by a Rogowski coil technique. The Faraday rotation technique measured the $16 \mathrm{MA}$ currents in these experiments with better than $1 \%$ precision.

In addition, six B-dot probes were fielded at equal angles around a circle in the powerflow channel outside the liner to measure the symmetry of the liner impact on the target cylinder. The B-dot probes measure the local Idot, which has a jump when the liner impacts the target cylinder. A high-pass filter allows one to measure this jump more accurately. From the relative timing of the jump signals, the offset of the liner axis and the circularity of liner are inferred.
\end{abstract}

\section{INTRODUCTION}

When liner implosion experiments are done on a pulsed power machine, a measurement of the current delivered to the liner is essential to know the driving conditions on the liner. On the Near Term Liner Experiments (NTLX) series of tests at Air Force Research Laboratory (AFRL), we used the Faraday rotation technique to measure this current. In addition, we fielded six B-dot detectors in the powerflow channel from which we could determine the impact time of the liner onto the target and measure the asymmetry of the collision.

\section{CURRENT MEASUREMENT}

The Faraday rotation system measures the rotation of linearly polarized light in a glass fiber exposed to a magnetic field [1]. When the glass fiber makes one complete circuit around a current path, the total rotation of the polarization is a function only of the encircled current, independent of the detailed shape of the fiber path [2]. The proportionality constant between rotation and current is the Verdet constant. Our sensing fiber was Corning's Flexcor single mode fiber. Its Verdet constant was measured by the National Institute of Standards and Technology to be $2.65 \pm 0.03 \mathrm{rad} / \mathrm{MA}$ at $826.0 \pm 0.5 \mathrm{~nm}$.

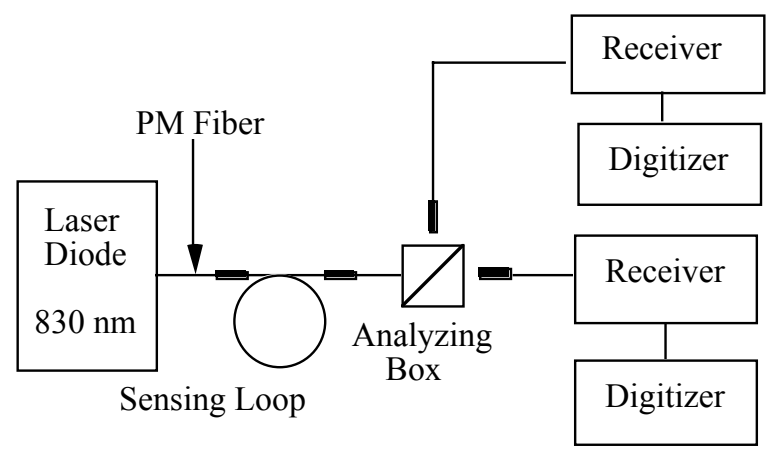

Figure 1. Schematic of the Faraday Rotation apparatus for measuring current.

Our fibers were located in the parallel-plate transmission line at a radius of 22.25 in. Two fibers were used to provide redundant measurements. Fig. 1 shows the setup for recording the signals from one fiber. Two signals are produced from the fiber by separating orthogonal polarizations. The resulting sine and cosine signals facilitate detection of current peaks.

The current measured for NTLX-2 is shown in Fig.2. The current measured by AFWL using a Rogowski coil is shown for comparison. The Rogowski current has been scaled by 1.09 to bring the peak values into agreement. All four Faraday currents and the scaled Rogowski overlay one another until about $17 \mu \mathrm{sec}$, then one fiber loses a fringe due to birefringence. This channel is offset by 1.18 MA for the remainder of the trace. Faraday fibers are sensitive to mechanical shocks so it is very useful to have a Rogowski measurement, especially for later times.

\footnotetext{
* Work supported by the US Department of Energy under contract W-7405-ENG-36 to Los Alamos National Laboratory, Los Alamos, NM, operated by the University of California. As an institution, LANL does not endorse the viewpoint of a publication or guarantee its technical correctness.

1. Sumner Associates, 2. Bechtel-Nevada, Los Alamos, 3. Bechtel-Nevada, Las Vegas, 4. NumerEx
} 


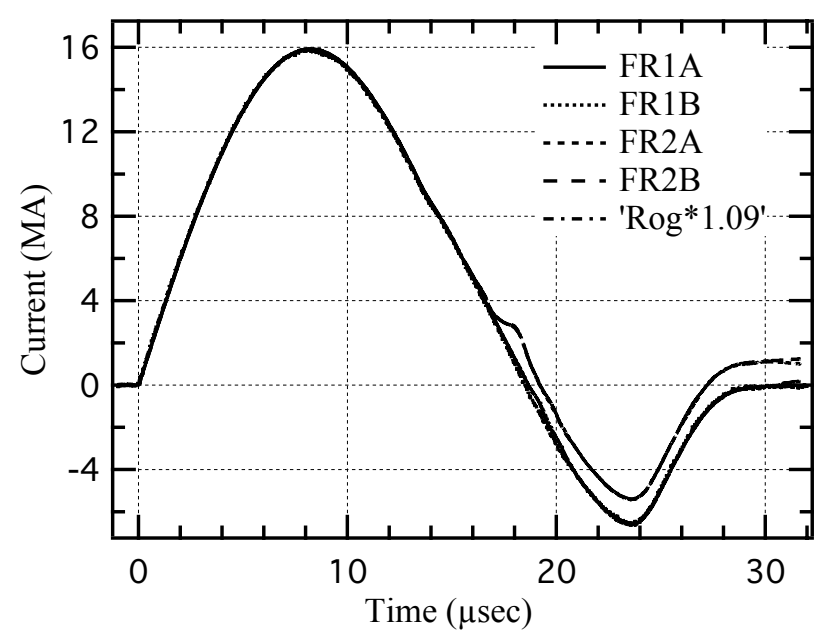

Figure 2. Four Faraday Rotation current measurements and a Rogowski current measurement (scaled by $9 \%$ ) on the NTLX-2 experiment.

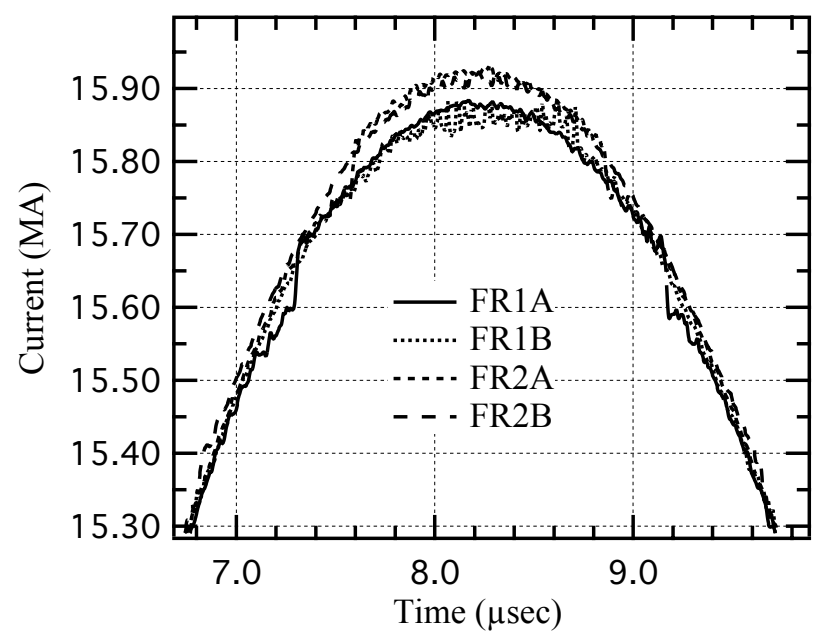

Figure 3. Faraday Rotation current traces expanded near peak current On the NTLX-2 experiment.

Figure 3 shows the Faraday Rotation measurements near peak on a sensitive scale to show the deviation obtained on NTLX-2. The total spread among the four Faraday channels is $80 \mathrm{kA}$ or about $0.5 \%$ of the current. Similar results were obtained for all four NTLX experiments. The peak currents measured were 16.0, 15.9, 15.74, and 15.76 MA for NTLX-1, 2, 3, and 4 respectively.

\section{SYMMETRY MEASUREMENT}

When impacting a liner onto a target, one would like to know when the impact occurred and how simultaneous the impact was in azimuth around the target. To measure these quantities, six B-dot detectors were fielded at the same radius and equally spaced outside the initial radius of the liner. Each B-dot sensor consisted of two wires, counter-wound on a small Teflon cylinder. The two output signals were recorded differentially to reduce common-mode noise.
A sample I-dot signal for NTLX-3 is shown in Fig. 4. Note the jump in the I-dot at about $14 \mu \mathrm{s}$. This jump is caused by the sudden change in the velocity of the liner outer surface. The jump time is delayed relative to target impact by the transit time of a shock wave through the liner. This delay is calculated to be $230 \mathrm{~ns}$ for the NTLX experiments.

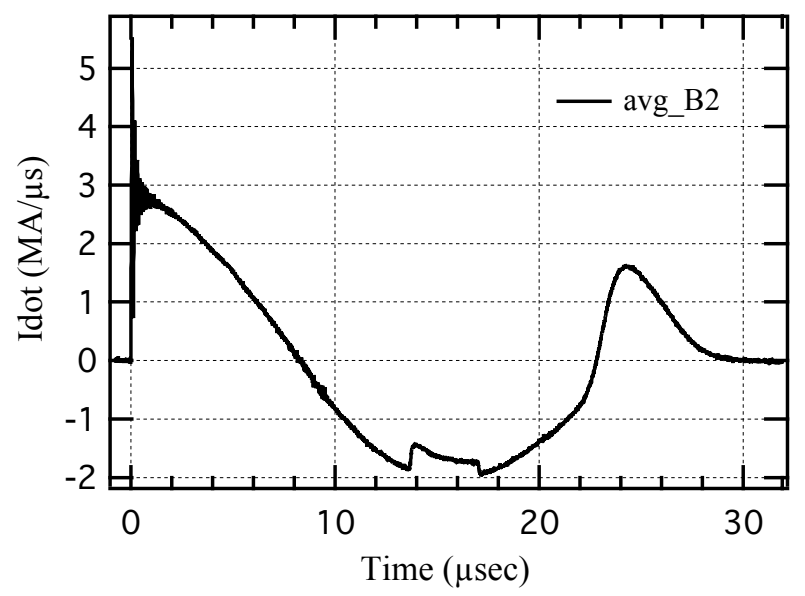

Figure 4. Time rate of change of the B-field for one of six symmetry probes on the NTLX-3 experiment.

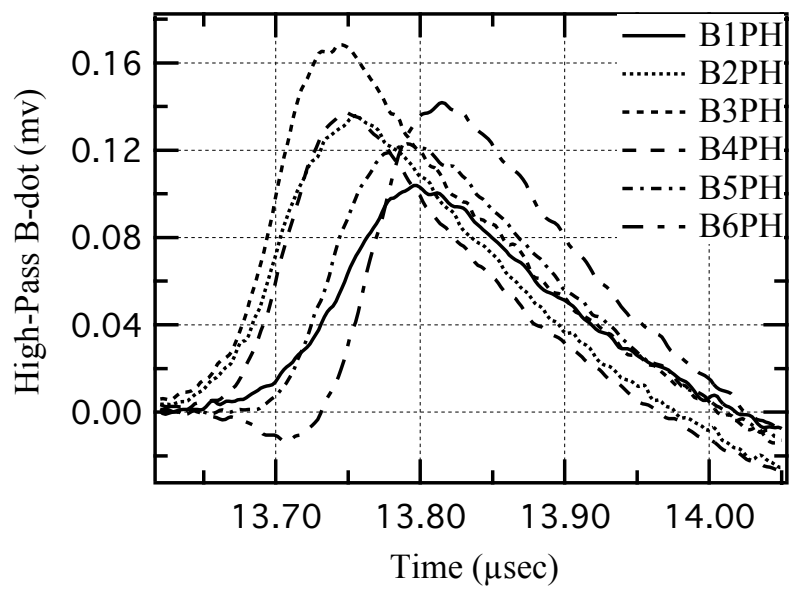

Figure 5. High-Pass filtered B-dot signals for the NTLX-3 experiment.

To get a better measurement of the jump, we split the B-dot signal and sent it through a high-pass filter to get rid of the large cosine signal. Then the recording sensitivity was increased. Fig. 5 shows the jump in the high-pass signals for the six B-dot detectors on NTLX-3. The relative timing of the six detectors is aligned accurately at the start of current flow. The difference in jump times shown in Fig. 5 results from small differences in the impact time around the azimuth of the target. To analyze this data we use the time at half-peak amplitude to represent the jump time. Averaging the six jump times gives an average jump time of $13.72+/-0.03 \mu \mathrm{s}$. Correcting for transit time, the inferred impact time is $13.49+/-0.03 \mu \mathrm{s}$. 
We measured the symmetry of the impact by multiplying the jump times (less an arbitrary offset time) by the sine and cosine of the angular position of the corresponding probe. The results are plotted on a $x-y$ plot as shown by the dots in Fig.6 for NTLX-3 data. We fit these times to an offset circle as shown by the solid curve in Fig. 6 . The radius of this is arbitrary, depending on the offset time subtracted from the measured impact times. The important parameters in Fig.6 are the deviation of the measured data from the ideal circle and the offset of the center of the circle from the zero of the coordinate axes.

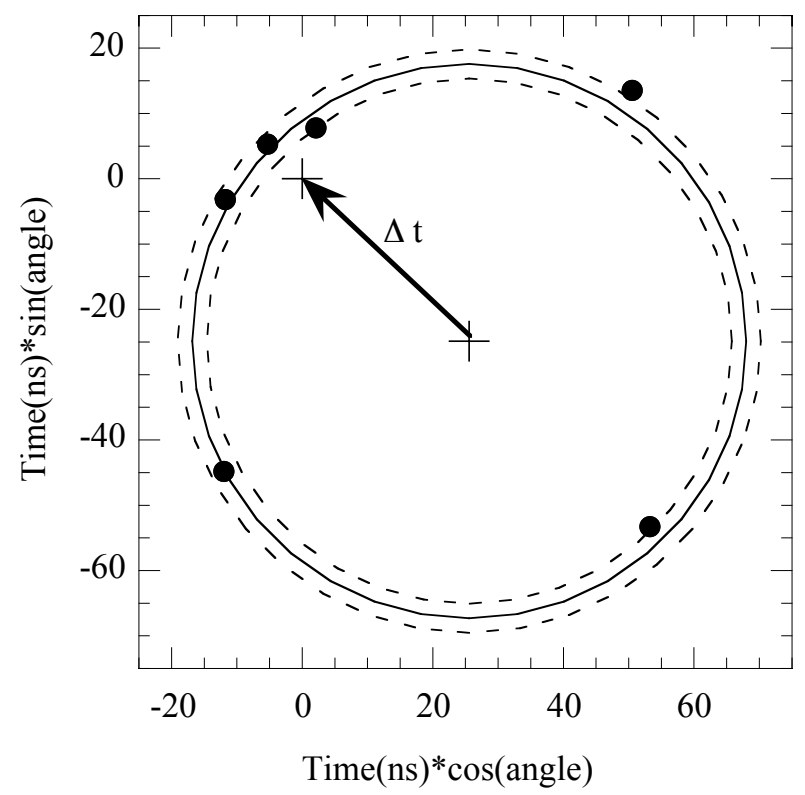

Figure 6. Plot of impact times fit to an offset circle for the NTLX-3 experiment.

The calculated $1 \sigma$ deviation of the measured jump times from the ideal circle is $2.2 \mathrm{~ns}$ for the NTLX-3 data shown in Fig. 6 . The $\pm 1 \sigma$ band is indicated by the dashed circles. Converting this time deviation to distance using the impact velocity $6.25 \mathrm{~mm} / \mu \mathrm{s}$ shows that the liner deviated from a perfect circle by $\pm 14 \mu \mathrm{m}$ at impact. This impact velocity was inferred from a comparison of calculation and measured impact time.

The amount of offset between the circle center and the coordinate origin at 0,0 in Fig. 6 is a measure of the physical offset between the center of the target and the axis of the liner at impact. The amount of offset $(\Delta t)$ for the NTLX-3 data of Fig. 6 is $35.7 \mathrm{~ns}$. Converting the offset time to distance using the impact velocity gives a physical offset of $223 \mu \mathrm{m}$. The direction of the offset relative to the target center is 120.8 degrees on the machine. (Zero degrees is north on the machine with angle increasing toward the west.) This is the location of the earliest impact of the liner onto the target.

The results of the symmetry measurements for all four NTLX experiments are summarized in the Table. Due to lack of room in the target cylinder, there were no independent measurements of impact time to confirm the B-dot measurement. To provide some indication of the accuracy of this method, the last two entries in the Table show a comparison between fiber optic pins and B-dot symmetry measurements on the MB-3 experiment on Pegasus. This comparison shows that the B-dot method can provide accurate impact information without the necessity of deploying diagnostics in the limited target volume.

\begin{tabular}{|c|c|c|c|c|}
\hline \multicolumn{5}{|c|}{ Table } \\
\multicolumn{5}{|c|}{$\begin{array}{c}\text { Summary of liner impact parameters for the NTLX } \\
\text { experiments as measured by the B-dot array. }\end{array}$} \\
\hline Shot \# & $\begin{array}{c}\Delta \mathrm{t} \\
(\mathrm{ns})\end{array}$ & $\begin{array}{c}\text { Direction } \\
(\text { deg. })\end{array}$ & $\begin{array}{c}\text { Offset } \Delta \mathrm{r} \\
(\mu \mathrm{m})\end{array}$ & $\begin{array}{c}\sigma \sigma \text { Dev. } \\
(\mu \mathrm{m})\end{array}$ \\
\hline NTLX-1 & 64.7 & 5.1 & 404 & 15.1 \\
\hline NTLX-2 & 24.35 & 126.1 & 152 & 2.3 \\
\hline NTLX-3 & 35.7 & 120.8 & 223 & 2.2 \\
\hline NTLX-4 & 16.0 & 16.9 & 100 & 5.3 \\
\hline $\begin{array}{c}\text { MB-3 } \\
\text { Pins }\end{array}$ & 51.7 & 110.9 & 207 & 1.3 \\
\hline $\begin{array}{c}\text { MB-3 } \\
\text { Bdots }\end{array}$ & 49.9 & 108.4 & 200 & 4.7 \\
\hline
\end{tabular}

\section{SUMMARY}

Current waveforms were measured for four liner implosion experiments using the Faraday method. The measured currents were used for post-shot analysis of liner dynamics. The calculated liner impact time was in excellent agreement with the impact time measured by the B-dot array. Rogowski current measurements confirmed the Faraday waveforms, but had a lower amplitude that was not consistent with the measured impact times.

An array of B-dot probes outside the initial position of the liner was used to measure parameters of the liner impact. Analysis of the B-dot data gave three parameters; average impact time, offset of the liner axis at impact and a measure of the circularity of the liner at impact.

\section{REFERENCES}

[1] L.R. Veeser, G. I. Chandler, and G. W. Day, "Fiber optic sensing of pulsed currents," Photonic: High Bandwidth Applications, James Chang, ed., (SPIE 648. Bellingham, WA, 1986). p.197

[2] Stokes, J. L., et al., "Precision Current Measurements on Pegasus II Using Faraday Rotation," in Tenth IEEE International Pulsed Power Conference (Albuquerque, NM), edited by W. Baker and G. Cooperstein, IEEE, Piscataway, NJ, 1995, Vol. 1, pp. 378-383 